\title{
Characteristics of Zanshin of Kendo Practitioners: Examination of the Relationship between Eye Blinking and CNV Resolution Time
}

\author{
Masayuki Hamaguchi', Hiroshi Asada', Yu Aramaki² and Kaoru Kitagawa ${ }^{2}$ \\ ${ }^{1}$ Faculty of Liberal Arts and Sciences, Osaka Prefecture University, 1-1 Gakuen-cho, Naka-ku, Sakai-shi, Osaka 599-8531, Japan \\ E-mail: hamaguti@las.osakafu-u.ac.jp \\ ${ }^{2}$ Laboratory for Exercise and Sports Physiology, School of Health and Sports Science, Chukyo University, \\ 101 Tokodachi, Kaizu-cho, Toyota-shi, Aichi, 470-0393, Japan \\ [Received March 7, 2014; Accepted July 23, 2014; Published online August 5, 2014]
}

\begin{abstract}
The purpose of this study was to uncover the characteristics of zanshin (spirit that remains after striking an opponent) of kendo practitioners. Kendo practitioners and non-kendo practitioners were compared from a unique perspective: the relationship between their eye blink control and the duration of their contingent negative variation $(\mathrm{CNV})$. The $\mathrm{CNV}$ is related to continuation of expectancy or attention.

The participants were divided into kendo and non-kendo groups. The participants performed discriminative stimulus tasks (Go and No-go button press tasks). The following were measured in these tasks: the area of the CNV plotted on a graph between 1800 and $2000 \mathrm{~ms}$ after the first light stimulus (S1); duration of the CNV after the second light stimulus (S2) (CNV resolution time); button-press reaction time in response to $\mathrm{S2}$; and the timing of the first eye blink between 1000 and $2000 \mathrm{~ms}$ after S2. Data analysis showed no significant differences in reaction time and the CNV area between the two groups. The CNV ended significantly later for the kendo group than for the non-kendo group in the Go task. Also, the kendo group blinked their eyes after S2 significantly later than the non-kendo group. There was therefore a significant positive correlation between the CNV resolution time and the timing of eye blinking. The study uncovered one of the characteristics of zanshin by indicating that the kendo group sustained their attention during internal information processing even after completion of the task.
\end{abstract}

Keywords: zanshin, kendo practitioner, eye blink, contingent negative variation (CNV), CNV resolution time

\section{Introduction}

Kendo is an athletic competition in which two players in protective equipment strike each other with a bamboo sword called shinai. In Kendo, referees determine the winner of the competition; they use flags to indicate that strikes meet the criteria for valid strikes called yuko datotsu which then determine the game result. Yuko datotsu, as defined in the All Japan Kendo Federation's rulebook called "The Regulations of Kendo Shiai and Shinpan,"*1 must meet all of 6 criteria. Five of these are: (1) high spirit, (2) correct posture, (3) striking with a specified part of the shinai (datotsu-bu), (4) striking of a specified target point (datotsu-bui), and (5) striking with the correct blade angle (hasuji). These criteria are related to datotsu or strike quality. The last 6th criterion is the presence of zanshin. Zanshin is a state in which a player stays on guard after executing a strike. One of the most distinct characteristics of kendo is that, unlike Western fencing, the yuko datotsu criteria includes the rather spiritual gesture of zanshin, which can only be present after execution of a strike, for determination of the match result. As a result of such inclusion of the spiritual criteria, and based on the belief that the match still continues after datotsu execution, kendo training always includes mental strength practice focused on sustaining attention on the opponent instead of dismissing the flow of attack due to the feeling that the match is finished. Due to the nature of zanshin, its presence/absence is determined by referees more 
subjectively than objectively. Therefore, identification of zanshin-related physiological responses that can serve as grounds for objective judgment should have significant meaning.

There have been studies on eye blinking to identify specific methods of sustaining attention. Baumstimler and Parrot (1971) for instance conducted an experiment in which the participants pressed a key in response to visual stimuli. They reported that the participants suppressed their eye blinking before they pressed the key but blinked their eyes repeatedly as soon as they pressed the key. According to Baumstimler, it was as if they were released from this need to suppress. Fukuda (2001) and Pivik and Dykman (2004) suggested in their studies that the time before post-task voluntary eye blinking was influenced by the level of attention to, response to, and cognition of the task. Also, Ishigaki (2005) studied eye blinking during table tennis rallies and while kendo player's face each other. The table tennis players were found to control their eye blinking more than usual during a one-minute rally, and the same eye blink control was observed in kendo practitioners while they stood facing each other with their shinai in contact. Furthermore, some studies reported that the level of eye blink control depended on the complexity of tasks or emotions towards the object (Fogarty and Stern, 1989; Goldstein et al., 1992; Ridder and Tomlinson, 1997). Thus eye blink control appeared to be dependent to some degree on intensity of attention to the object and continuity of such attention.

Meanwhile, Hamaguchi et al. (2012) compared between kendo practitioners and non-kendo practitioners the timing of their eye blinking in Go and No-go tasks using LEDs by recording their electrooculogram (EOG). The study showed that, the kendo practitioners continued to control their eye blinking significantly longer than the non-kendo practitioners even after the task was completed. Also, the kendo practitioners with higher competitive abilities exhibited significantly longer eye blink control. Hamaguchi et al. reported that these results may be attributable to the kendo practitioners' zanshin, an attempt unique to kendo to stay on guard even after datotsu execution. Their long-term everyday zanshin practice may have influenced their responses to stimuli in their daily non-kendo activities.

In connection with attention and its sustainment, the authors of this paper focused on one of the com- ponents of the event-related potential (ERP) called contingent negative variation $(\mathrm{CNV})$, which indicates the relationship between a motor function and brain information processing. According to Walter et al. (1964), the CNV is a negative potential that appears between a warning stimulus (S1) and imperative stimulus (S2). S2 is presented a certain amount of time after presentation of S1 and is also related to psychological factors such as expectancy, prediction, and attention (Tecce, 1972). The CNV consists mainly of early and late components. Hillman et al. (2000) and Van Boxtel and Brunia (1994) reported that, while the early component of the CNV reflected an orienting response to $\mathrm{S} 1$ and arousal level in response to $\mathrm{S} 1$, the late component of the $\mathrm{CNV}$ reflected both motor readiness and non-motor components. Note that the non-motor components included expectancy, prediction, and attention (Hillman et al., 2000; Van Boxtel and Brunia, 1994). Furthermore, both Brunia and Vingerhoets (1980) and Wascher et al. (1996) observed that shortened response time and an increase in the amplitude of the late CNV component occurred simultaneously in their studies which examined the relationship between the late $\mathrm{CNV}$ component and motor responses.

Meanwhile, the process in which the CNV returns to the baseline after $\mathrm{S} 2$ (CNV resolution process) is also believed to reflect brain activities. Kakigi (1977) argued that the CNV resolution process was caused by a psychological factor associated with completion of a task. Meanwhile, Iwanaga et al. (2002) reported that the necessity of stimulus discrimination at S2 influenced the CNV resolution time. Kotani et al. (2009) and Chwilla and Brunia (1991) reported that their study participants exhibited an increase in the negative potential immediately before they received a feedback stimulus (S3) as an indicator of how well they performed in earlier motor response tasks. If this situation is applied to kendo, possibility of a next opponent's potential attack after his datotsu would be the feedback stimulus S3, and the psychological factor represented as sustained attention may be exhibited as zanshin. Although comparison of CNV waveforms between kendo and non-kendo practitioners does not use any feedback criteria as used by Kotani et al. (2009) and Chwilla and Brunia (1991), it may still show the influence of zanshin training in the $\mathrm{CNV}$ waveforms that maintain their amplitude and then return to the baseline after S2. 
This study compared kendo practitioners and non-kendo practitioners in terms of the relationship between the CNV resolution time and the first eye blinking observed after S2. The objective of this study was to gain insight into a specific method to train the mind by uncovering the characteristics of zanshin of kendo practitioners, which is one of necessary parts of yuko datotsu and also was associated with sustaining of attention, while referring to the comparison result.

For the purpose of this study, discriminative visual stimulus tasks (Go/No-go button press tasks) were administered. A discriminative visual stimulus task is a widely used method to examine the neural basis of execution or withholding of a motor response.

\section{Method}

\subsection{Participants}

The participants were divided into two groups: kendo and non-kendo groups. The kendo group consisted of 7 male undergraduate students who had 10 years of kendo experience or longer and were members of a kendo club at the time of the study $(21.3 \pm 1.5$ years old). They were all 3-dan (rank) practitioners. The non-kendo group consisted of 9 male undergraduate students who were not members of any athletic sports club at the time of the study and had no regular exercise habits $(22.4 \pm 3.3$ years old). None of the participants had a history of neurological disease. They were all right handed. For ethical considerations, the objective and the significance of the experiment were provided in written form as well as orally explained to the participants prior to the experiment in line with the World Medical Assembly's Declaration of Helsinki (Kuroyanagi, 2009). The experiment was conducted after the experimenters obtained written consent from the participants for voluntary participation in the experiment.

\subsection{Experiment}

A classroom desk and a chair were placed in a simple shielded room. Each participant sat comfortably on the chair. Next, he was asked to place his right hand on the Styrofoam box on the desk and then place the right index finger on the button placed at the center of the top of the box. There were 3 LED lamps (1 yellow lamp between 2 red lamps, each having a $0.5 \mathrm{~cm}$ diameter) installed horizontally against a black curtain 1 meter from the participant (left and right viewing angle: 0.57 degrees each). The participants confirmed that they were able to see the light stimuli without using glasses or contact lenses and then performed the tasks without wearing them. In the experiment, the participants were first told to gaze at the yellow lamp (1st stimulus: S1) that randomly flashed in 5 to 7 second intervals. Then, 2 seconds after S1, either the left or right red lamp randomly lit up with $50 \%$ probability (2nd stimulus: RS2 for the right lamp and LS2 for the left lamp). The lamps stayed lit for $150 \mathrm{~ms}$. In the Go task, the participants were instructed to press the button with the right index finger as fast as possible when they saw the 2nd stimulus. The experiment consisted of 4 types of task: the right Go task requiring a button press response when the right red light turned on; the left Go task requiring a button press response when the left red light turned on; the right No-go task requiring withholding of a button press response when the right red light turned on; and the left No-go task requiring withholding of a button press response when the left red light turned on. The experiment was counterbalanced among the participants in order to avoid the effect of learning or being too accustomed to the task. The time of brain wave measurement was consistent throughout the experiment for each participant in order to avoid the influence of daily fluctuation.

\subsection{Measurement}

A scalp electrode with $10 \mathrm{~K} \Omega$ impedance or lower was attached to the vertex $(\mathrm{Cz})$ site of the participants in accordance with the International 10-20 system, and brain waveforms were recorded using unipolar leads with linked earlobe electrodes as a reference. The CNV sampling frequency was 500 $\mathrm{Hz}$, time constant was 2.0, and high-cut frequency was $30 \mathrm{~Hz}$ (EEG9100 by Nihon Kohden Corporation). At the same time, stimulus signals S1, LS2, and RS2 as well as the button-press response were measured and recorded. In addition to the brain waves, both horizontal and vertical eye movements were recorded (EOG). After the measurement, a Kissei Comtec EPLYZER II was used off-line to obtain the grand average of the post-S1 CNV 
waveform for both Go and No-go tasks. The CNV was measured for the 3-minute resting state and 3minute tasks with a 2 -minute break in between. In each task, the participants performed 12 sets of 25 Go and No-go random trials. Note that the data affected by artifacts, which are potentials other than brain potentials, data containing a large amount of noise, and incorrect responses to the stimuli $(1.5 \%$ of all trials) were excluded from analysis. As a result, data obtained from 260 trials (130 Go and 130 No-go trials) was analyzed.

Reaction time to the Go stimulus was examined for all participants. In this study, reaction time was the time between the Go stimulus and the button press response. As described earlier, incorrect responses were first excluded from the data, and then the average reaction time was used in analysis.

The CNV waveform of a total of $5000 \mathrm{~ms}$, from $1000 \mathrm{~ms}$ before S1 to $2000 \mathrm{~ms}$ after S2, was analyzed. The CNV waveform between $1000 \mathrm{~ms}$ before S1 and S1 was obtained as the baseline for each participant, and its grand average was calculated for each task type. The CNV area was obtained by calculating the integral of the late $\mathrm{CNV}$ waveform, which appeared between 1800 and $2000 \mathrm{~ms}$ after S1. The CNV resolution time was a period of time between the positive shift of the CNV after $\mathrm{S} 2$ and the $\mathrm{CNV}$ waveform touching the baseline. In accordance with the study by Hamaguchi et al., the first eye blinking after $\mathrm{S} 2$ was obtained by averaging the time of the first eye blinking that occurred between 1000 and $2000 \mathrm{~ms}$ after S2.

\subsection{Statistical analysis}

Two-way analysis of variance (ANOVA) with repeated measures was conducted for the CNV area, $\mathrm{CNV}$ resolution time, and the first post-S2 eye blinking. Kendo experience (kendo or non-kendo group) and task type (Go or No-go) were used as independent variables. The $t$-test was conducted to compare the reaction time between the kendo and non-kendo groups. The relationship between the CNV resolution time and the first post-S2 eye blinking was analyzed using Pearson's product-moment correlation coefficient. Statistics software IBM SPSS Statistics 22 and js-STAR 2012 (2.0.6j) was used in analysis.

\section{Result}

\subsection{Grand average of the CNV waveform and the CNV area}

Figure 1 shows the grand average of the $\mathrm{CNV}$

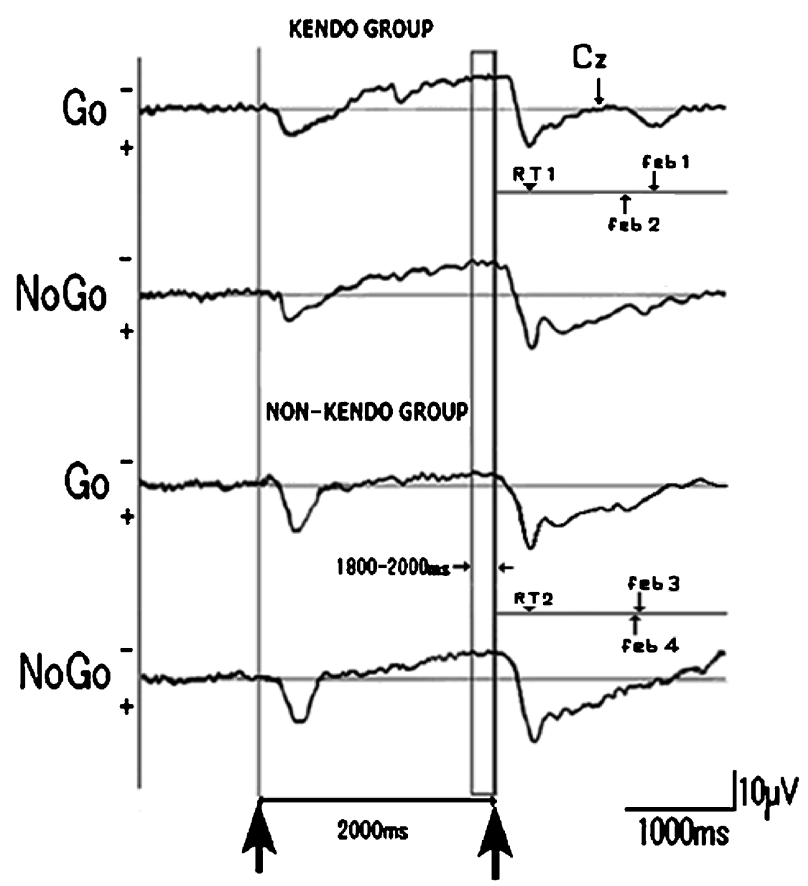

Figure 1 GA (Grand Average) of the CNV waveforms of the Kendo and Non-Kendo groups

$※$ RT1: reaction time of the kendo group

$※ \mathrm{RT} 2$ : reaction time of the non-kendo group

$※$ feb1: the first eye blinking by the kendo group in the Go task

$※$ feb2: the first eye blinking by the kendo group in the No-go task

$※$ feb3: the first eye blinking by the non-kendo group in the Go task

$※$ feb4: the first eye blinking by the non-kendo group in the Nogo task

Table 1 Inter-group comparison of CNV area

\begin{tabular}{|c|c|c|c|c|c|c|c|}
\hline \multirow{3}{*}{$\begin{array}{c}\text { Kendo experience } \\
\text { Task type }\end{array}$} & \multicolumn{4}{|c|}{ CNV Area between 1800 and $2000 \mathrm{~ms}$ after S1 } & \multicolumn{3}{|c|}{$\begin{array}{r}\text { Two-way ANOVA } \\
F \text {-value }\end{array}$} \\
\hline & Kendo gr & oup $(n=7)$ & Non-kendo & roup $(n=9)$ & Kendo experience (A) & 0.04 & $n s$ \\
\hline & $\begin{array}{c}\text { Go } \\
1092.2(1243.0)\end{array}$ & $\begin{array}{c}\text { No-go } \\
1082.1(1086.3)\end{array}$ & $\begin{array}{c}\text { Go } \\
936.3(747.5)\end{array}$ & $\begin{array}{c}\text { No-go } \\
1046.6(752.2)\end{array}$ & $\begin{array}{l}\text { Task type }(\mathrm{B}) \\
(\mathrm{A} \times \mathrm{B})\end{array}$ & $\begin{array}{l}0.18 \\
0.26\end{array}$ & $\begin{array}{l}n s \\
n s\end{array}$ \\
\hline
\end{tabular}

$n s:$ not significant

Indicated in the table with $\mathrm{M}$ (SD) 
waveform measured in the $\mathrm{Cz}$ area, reaction time in response to $\mathrm{S} 2$, and the timing of the first post-S2 eye blinking for the kendo and non-kendo groups in the Go and No-go tasks. The area of the late CNV waveform between 1800 and $2000 \mathrm{~ms}$ after S1 was obtained and compared between the kendo and nonkendo groups and also between the Go and No-go tasks. As Table 1 shows, the main effect of kendo experience $(F(1,14)=0.04$, ns), main effect of the task type $(\mathrm{F}(1,14)=0.18, \mathrm{~ns})$, and interaction effect of these two variables $(\mathrm{F}((1,14)=0.26, \mathrm{~ns})$ were not significant.

\subsection{Reaction time}

Reaction time was compared between the kendo and non-kendo groups using the $t$-test. The result indicated no significant difference $(\mathrm{t}(14)=0.58$, ns) between the two groups (Table 2).

Table 2 Inter-group comparison of CNV reaction time

\begin{tabular}{|c|c|c|c|c|}
\hline & \multicolumn{2}{|c|}{ Reaction time (ms) } & $t$-value & $p$-value \\
\hline $\begin{array}{c}\text { Kendo } \\
\text { experience }\end{array}$ & $\begin{array}{l}\text { Kendo group } \\
(\mathrm{n}=7)\end{array}$ & $\begin{array}{l}\text { Non-kendo } \\
\text { group }(n=9)\end{array}$ & 0.58 & $n s$ \\
\hline
\end{tabular}

student's $t$-test, $n s$ : not significant Indicated in the table with M (SD)

\subsection{CNV resolution time}

Two-way analysis of variance (ANOVA) with repeated measures was conducted for the CNV resolution time. Kendo experience (kendo or non-kendo group) and task type (Go or No-go) were used as independent variables. As Table 3 shows, the main effects of kendo experience (kendo and non-kendo groups: $\mathrm{F}(1,14)=0.17, \mathrm{~ns})$ and task type (Go and No-go tasks: $F(1,14)=2.99$, ns) were not significant . The interaction effect of these two variables however was significant $(\mathrm{F}(1,14)=33.58$, $\mathrm{p}<0.01)$. The simple main effect of each element was then analyzed. From the kendo experience perspective, the CNV resolution time was significantly longer $(F(1,14)=9.93, p<0.01)$ for the kendo group than the non-kendo group in the Go task. There were however no significant differences between the groups in the No-go task $(\mathrm{F}(1,14)=2.44, \mathrm{~ns})$.

From the task type perspective, the CNV resolution time was significantly longer in the Go task than in the No-go task for the kendo group $(F(1,14)=$ $8.26, \mathrm{p}<0.05)$. On the other hand, the CNV resolution time was significantly longer in the No-go task than in the Go task for the non-kendo group $(\mathrm{F}(1,14)=28.31, \mathrm{p}<0.01)$.

Table 3 Inter-group comparison of CNV resolution time

\begin{tabular}{|c|c|c|c|c|c|c|c|c|}
\hline & \multicolumn{4}{|c|}{ CNV resolution time (ms) } & \multicolumn{4}{|c|}{$\begin{array}{l}\text { Two-way ANOVA } \\
\qquad F \text {-value } p \text {-value }\end{array}$} \\
\hline Kendo experience & Kendo gro & up $(n=7)$ & Non-kendo & group $(n=9)$ & Kendo experience (A) & 0.17 & $n s$ & \\
\hline Task type & Go & No-go & Go & No-go & Task type (B) & 2.99 & $n s$ & \\
\hline$\overline{\mathrm{X}}(\mathrm{SD})$ & $195.7(41.9)$ & $157.7(43.5)$ & $132.1(38.6)$ & $202.4(65.0)$ & $(\mathrm{A} \times \mathrm{B})$ & 33.58 & $* *$ & $※ 1$ \\
\hline
\end{tabular}

Table 4 Inter-group comparison of timing of the first post-S2 eye blinking

\begin{tabular}{|c|c|c|c|c|c|c|c|}
\hline & \multirow{2}{*}{\multicolumn{4}{|c|}{ First eye blinking between 1000 and $2000 \mathrm{~ms}$ after S2 (ms) }} & \multicolumn{3}{|c|}{ Two-way ANOVA } \\
\hline & & & & & $F$-value & $p$-value & Multiple comparison \\
\hline $\begin{array}{l}\text { Kendo } \\
\text { experience }\end{array}$ & Kendo group $(n=7)$ & \multicolumn{2}{|c|}{ Non-kendo group $(\mathrm{n}=9)$} & \multirow{3}{*}{$\begin{array}{c}\text { Kendo experience }(\mathrm{A}) \\
\text { Task type }(\mathrm{B}) \\
(\mathrm{A} \times \mathrm{B})\end{array}$} & 31.40 & $* *$ & \multirow[t]{2}{*}{$\begin{array}{l}\text { Kendo group }> \\
\text { non-kendo group }\end{array}$} \\
\hline Task type & No-go & Go & No-go & & 0.48 & $n s$ & \\
\hline$\overline{\mathrm{X}}(\mathrm{SD})$ & $1335.3(43.8) 1249.3(98.9)$ & $1108.4(63.9)$ & $1231.7(60.9)$ & & 15.10 & $* *$ & $※ 1$ \\
\hline
\end{tabular}

$※ 1$ Simple main effect of $\mathrm{A} \times \mathrm{B}$ interaction

- Kendo experience (Go): $F=64.28^{* *}$ [kendo group $>$ non-kendo group]; kendo experience (No-go): $F=0.19$, $n s$

- Task type (kendo group): $F=5.10^{*}$ [Go $>$ No-go]; task type (non-kendo group): $F=10.48^{* *}$ [Go $<$ No-go] 


\subsection{Timing of the first post-S2 eye blinking}

Two-way analysis of variance (ANOVA) with repeated measures was conducted for timing of the first post-S2 eye blinking using kendo experience (kendo or non-kendo group) and task type (Go or No-go) as independent variables. As Table 4 shows, the main effect of kendo experience (kendo and nonkendo groups: $F(1,14)=31.40, p<0.01)$ was significant. There was no significant difference between the Go and No-go tasks $(F(1,14)=0.48, n s)$. The interaction effect of the two variables however was significant $(F(1,14)=15.10, p<0.01)$. Furthermore, the simple main effect of each element was analyzed. As a result, the first post-S2 eye blinking occurred significantly later $(\mathrm{F}(1,14)=64.28, \mathrm{p}<0.01)$ for the kendo group than the non-kendo group in the Go task.

There were however no significant differences between the two groups in the No-go task $(F(1,14)=$ $0.19, \mathrm{~ns})$.

From the task type perspective, the first post-S2 eye blinking occurred significantly later in the Go task than in the No-go task for the kendo group $(F(1,14)=5.10, p<0.05)$. On the other hand, the first post-S2 eye blinking was significantly later in the No-go task than in the Go task for the nonkendo group $(F(1,14)=10.48, p<0.01)$.

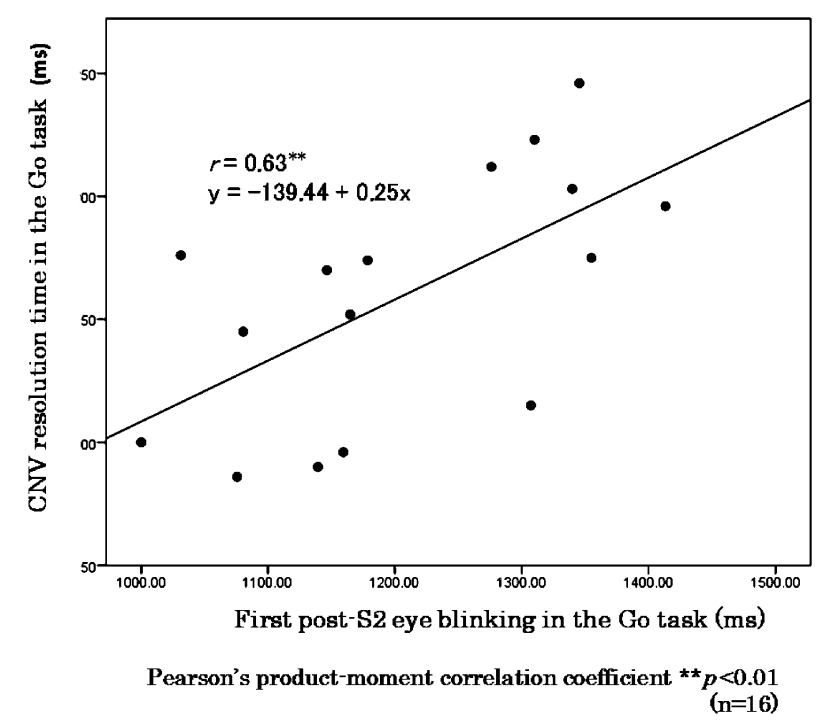

Figure 2 Relationship between CNV resolution time and the first eye blinking after S2

\subsection{Relationship between $\mathrm{CNV}$ resolution time and the first eye blinking after S2}

The CNV resolution time and the first eye blinking after S2 had similar statistical results. Calculation of Pearson's product-moment correlation coefficient resulted in a significant positive correlation $(r=0.63$, $\mathrm{p}<0.01$ ) between these two variables. Figure 2 provides a scatter diagram of the correlation.

\section{Discussion}

With the objective of uncovering the characteristics of zanshin, which was associated with sustained attention by kendo practitioners, the authors of this study compared kendo and non-kendo practitioners regarding the area of the late $\mathrm{CNV}$ waveform, reaction time in response to $\mathrm{S} 2$, post-S2 CNV resolution time, and the first eye blinking after S2.

The two participant groups indicated no significant differences in the late CNV waveform area and reaction time. According to Van Boxtel and Böcker (2004), the late CNV waveform included non-motor components, and it was therefore important to record electromyograms to ensure the myoelectric output is constant across the experimental conditions. In this study, the kendo and nonkendo groups indicated no significant difference in $\mathrm{CNV}$ waveform area, and this may be attributable to great variation in the myoelectric output. Great variation in the myoelectric output would cause the motor components in the late $\mathrm{CNV}$ to vary greatly across the experimental conditions. This in turn would prevent the inter-group differences in perceived readiness (non-motor components) from being reflected in the statistical result. The study therefore suggested the importance of recording electromyograms to control myoelectric output in future CNV measurement.

As for the CNV resolution time, Iwanaga et al. (2002) reported that it was significantly shorter in discriminative motor tasks than in simple motor tasks. In this study, too, the CNV resolution time was significantly shorter in the Go task than the Nogo task for the non-kendo group. In addition, Imashioya et al. (1987) argued that more psychologically demanding tasks resulted in a shorter CNV resolution time and that the $\mathrm{CNV}$ resolution process therefore was influenced by the feeling associated with completion of tasks. This study also found that, for 
the non-kendo group, the more psychologically demanding task (Go task) resulted in a shorter CNV resolution time (132.1 $\pm 38.6 \mathrm{~ms}$ for the Go task and $202.4 \pm 65.0 \mathrm{~ms}$ for the No-go task). This study therefore reached the same results as the studies by Iwanaga et al. and Imashioya et al. suggesting that the $\mathrm{CNV}$ resolution time was influenced by the feeling of completion of task. Contrary to the case of the non-kendo group, however, the CNV resolution time was significantly longer in the Go task for the kendo-group (195.7 $\pm 41.9 \mathrm{~ms}$ for the Go task and $157.7 \pm 43.5 \mathrm{~ms}$ for the No-go task). It is suspected that the participants in the kendo group sustained their attention even after S2 in the Go task. Matsumoto and Sakuma (2006) examined the CNV and post-imperative negative variation (PINV) waveforms of the participants under stress in the competitive button pressing game situation and reported that from the onset of the imperative stimulus many of the attached electrodes detected the PINV remaining negative until the game result was announced. This may therefore explain why the kendo group in this study showed a longer post-S2 $\mathrm{CNV}$ resolution time than the non-kendo group; Although this experiment was not conducted in a competitive setting, the participants' expectation for the result of pressing the button as quickly as possible was reflected in their $\mathrm{CNV}$ waveform. Meanwhile, the first eye blinking after S2 in the Go task was significantly later for the kendo group. This result agreed with the study by Hamaguchi et al. (2012), suggesting that the kendo group controlled their eye blinking. Stern et al. (1984) reported that eye blinking could serve as a physiological indicator of psychological processing, including expectancy and attention. Nakano et al. (2012) reported that, based on their study in which participants' eye blinking and brain activities were measured while they were watching a movie, eye blinking caused reduction of attention-related neural network activities. This result suggests that if there is a long time between the onset of action and eye blinking, attention is sustained for a long time accordingly. In the study for this paper, the kendo group maintained the CNV waveform amplitude for a longer period of time after the Go task and recorded a longer period of time between the onset of the Go-task and the first eye blinking. The study further found that the timing of the first post-S2 eye blinking and the post-S2 CNV resolution time had a positive correlation. These results suggest that the kendo group sustained their attention even after the Go-task was completed and allow for speculation that the zanshin custom of long-time kendo practitioners is generalized to general, non-kendo tasks.

Based on the above, zanshin in kendo can be defined as a behavior to sustain attention after an action, and both CNV resolution time and the time of first post-S2 eye blinking may serve as a physiological indicator of zanshin.

\section{Conclusion}

As a result of the study, the characteristics of zanshin associated with sustaining of attention as indicated in the CNV resolution time and the first post$\mathrm{S} 2$ eye blinking can be summarized as follows.

In the Go task, the post-S2 CNV resolution time was significantly longer for the kendo group than for the non-kendo group.

The kendo group also showed that their first postS2 eye blinking was significantly later than the nonkendo group. Since there was a positive correlation between the post-S2 CNV resolution time and the timing of the first eye blinking, the kendo group seemed to have sustained their attention after completing the task.

The study therefore uncovered some of the characteristics of zanshin of kendo practitioners from the perspective of the relationship between the $\mathrm{CNV}$ resolution time and the timing of the first postS2 eye blinking.

More specifically, the study suggested that generation of eye blinking after a reaction was controlled as a result of practicing the psychological aspect of kendo.

\section{Note}

*1 All Japan Kendo Federation. ("The Regulations of Kendo Shiai and Shinpan. Part1 Shiai, Chapter2 Shiai, Section2 Yukodatotsu, Article12."' published 1995)

\section{References}

Baumstimler, Y., and Parrot, J. (1971). Stimulus generalization and spontaneous blinking in man involved in a voluntary activity. J. Exp. Psychol., 88: 95-102.

Brunia, C. H., and Vingerhoets, A. J. (1980). CNV and EMG preceding a plantar flexion of the foot. Biol. Psychol., 11: 181-191.

Chwilla, D. J., and Brunia, C. H. (1991). Event-related potentials to different feedback stimuli. Psychophysiol., 28: 123-132. 
Fogarty, C., and Stern, J. A. (1989). Eye movements and blinks: their relationship to higher cognitive processes. Int. J. Psychophysiol., 8: 35-42.

Fukuda, K. (2001). Eye blinks: new indices for the detection of deception. Int. J. Psychophysiol., 40: 239-245.

Goldstein, R., Bauer, L. O., and Stern, J. A. (1992). Effect of task difficulty and interstimulus interval on blink parameters. Int. J. Psychophysiol., 13: 111-117.

Hamaguchi, M., Asada, H., and Kitagawa, K. (2012). A Study of Eyeblinking and Attention Concentration in Kendo Practitioners. Japan J Phys. Educ. Hlth. Sport Sci, 57: 119-127. (in Japanese)

Hillman, C. H., Apparies, R. J., and Hatfield, B. D. (2000). Motor and non-motor event-related potentials during a complex processing task. Psychophysiol., 37: 731-736.

Imashioya, H., Dollins, A. B., and Kakigi, S. (1987). Motor Response Information Influence on CNV Shape and Resolution Time. The Pavlov J Biol. Sci, 22: 1-6.

Ishigaki, H. (2005). The eyeblinks during rally of the table tennis and confrontation of the kendo. AITARA, 40(B): 267-268. (in Japanese)

Iwanaga, R., Misaki, K., Nishihira, Y., Hatta, A., and Fumoto, M. (2002). Effect of Motor Preparation, Motor Response and Stimulus Discrimination on Contingent Negative Variation (CNV) Resolution Processes. J. Exercise Sports Physiol., 9: 93-100. (in Japanese)

Kakigi, S. (1977). Slow Brain Potentials and Psychological Factors. Studies in the Humanities and Sci. Hiroshima Shudo Univ., 18: 27-61. (in Japanese)

Kotani, Y., Ohgami, Y., Kuramoto, Y., Tsukamoto, T., Inoue, Y., and Aihara, Y. (2009). The role of the right anterior insular cortex in the right hemisphere preponderance of stimuluspreceding negativity (SPN): an fMRI study. Neurosci. Lett., 450: 75-79.

Kuroyanagi, T. (2009). Considering the 2008 Revision of the WMA Declaration of Helsinki. JMAJ, 138: 752-769. (in Japanese)

Matsumoto, S., and Sakuma, H. (2006). CNV and PINV under Stress of Competition. JJBR, 33: 17-24. (in Japanese)

Nakano, T., Kato, M., Morito, Y., Itoi, S., and Kitazawa, S. (2012). Blink-related momentary activation of the default mode network while viewing videos. Proceedings of the National Academy of Sciences of the United States of America, 110: 702-706.

Pivik, R. T., and Dykman, R. A. (2004). Endogenous eye blinks in preadolescents: relationship to information processing and performance. Biol. Psychol., 66: 191-219.

Ridder, W. H. 3rd., and Tomlinson, A. (1997). A comparison of saccadic and blink suppression in normal observers. Vision Res., 37: 3171-3179.
Stern, J. A., Walrath, L. C., and Goldstein, R. (1984). The Endogenous Eyeblink. Psychophysiol., 21: 22-33.

Tecce, J. J. (1972). Contingent Negative Variation (CNV) and Psychological Processes in man. Psychol. Bull., 77: 73-108.

Van Boxtel, G. J. M., and Brunia, C. H. (1994). Motor and non-motor components of the Contingent negative variation. Int. J. Psychophysiol., 17: 269-279.

Van Boxtel, G. J. M., and Böcker, K. B. E. (2004). Cortical Measures of Anticipation. J. Psychophysiol., 18: 61-76.

Walter, W. G., Cooper, R., Aldridge, V. J., McCallum, W. C., and Winter, A. L. (1964). Contingent negative variation: an electric sign of sensorimotor association and expectancy in the human brain. Nature, 203: 380-384.

Wascher, E., Verleger, R., Jaskowski, P., and Wauschkuhm, B. (1996). Preparation for action: an ERP study about two tasks provoking variability in response speed. Psychophysiol., 33: 262-272.

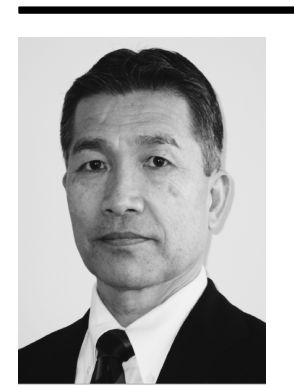

Name:

Masayuki Hamaguchi

\section{Affiliation:}

Faculty of Liberal Arts and Sciences, Osaka Prefecture University

Address:

1-1 Gakuen-cho, Naka-ku, Sakai-shi, Osaka 599-8531, Japan

Brief Biographical History:

-1977-1979 Master's Program in Health and Sports Science, Chukyo University

-1983-current Faculty of Liberal Arts and Sciences, Osaka Prefecture University

\section{Main Works:}

-Hamaguchi M, Asada H, Kitagawa K. (2012) A Study of Eyeblinking and Attention Concentration in Kendo Practitioners. Japan J Phys. Educ. Hlth. Sport Sci, 57:119-127.(in Japanese) Membership in Leaned Societies:

-Japan Society of Physical Education, Health and Sport Sciences

-The Japanese Society of Physical Fitness and Sports Medicine

- Japanese Society of Education and Health Science

- Japanese Academy of Budo

-The Japanese Society of Nutrition and Dietetics 